\title{
Analysis of DNA equilibrium configuration under interfacial traction
}

\author{
Y.Z. Wang ${ }^{1,2}$, Q.C. Zhang ${ }^{1,3}$, W. Wang ${ }^{1,3}$ and Y.W. Yang ${ }^{2}$ \\ ${ }^{1}$ School of Mechanical Engineering, Tianjin University, Tianjin, China \\ ${ }^{2}$ School of Mathematics and Statistics, Anyang Normal University, \\ Anyang, China \\ ${ }^{3}$ Tianjin Key Laboratory of Nonlinear Dynamics and Control, Tianjin, China
}

Corresponding authors: Y.Z. Wang / Q.C. Zhang

E-mail: wangyongzhao1987@126.com / qzhang@tju.edu.cn

Genet. Mol. Res. 15 (2): gmr.15028651

Received March 23, 2016

Accepted April 11, 2016

Published May 13, 2016

DOI http://dx.doi.org/10.4238/gmr.15028651

\begin{abstract}
Kirchhoff thin elastic rod models are important in the study of the mechanism determining the configurations of flexible structures not only at the macroscopic but also at the microscopic scale. In this study, the energy balance method has been well applied to analyze the configuration of a DNA elastic rod in the presence of interfacial traction. An approximate solution for the shape equations has been obtained, and the relationship between the interfacial factor and the configuration of the DNA segment is derived. The results may provide an explanation for the onset of the formation of kinks in DNA when immersed in a solution.
\end{abstract}

Key words: Thin elastic rod; Equilibrium configuration; Interfacial traction; Energy balance method; Kink; DNA 


\section{INTRODUCTION}

The chromosome is the main carrier of genetic material in living cells, and is a combination of DNA and proteins. The DNA is a long polymer made of hundreds of millions of nucleotides and is the carrier of hereditary information. Changes in the conformational features of DNA may affect many biological processes such as the replication and transcription of DNA, protein synthesis (Halford and Marko, 2004), and protein transport (Elf et al., 2007). The misfolding of DNA can also cause many illnesses, paroxysmal nocturnal hemoglobinuria (PNH) being one of them. In previous literature (Yavari, 2014), different filamentary structures, not only at the macroscopic but also at the microcosmic scale, have been regarded as thin elastic rods with characteristic elastic behavior. In recent years, a lot of models have been established for the study of microcosmic molecular chain structures, such as the general equilibrium configuration equations of thin elastic rods, which can be traced back to the 1850s; the wormlike chain model (Goldstein and Langer, 1995), which characterized the molecular chain structure under small external forces; and the wormlike rod chain model (Bouchiat and Mezard, 1998). In this study, a thin elastic rod in the presence of interfacial traction is used to study the geometric configurations of DNA in intracellular solutions at the microscopic scale.

Theoretical analysis models derived from the classical elasticity theory have no spatial/ temporal limitations and have widely used to study DNA reconfiguration. Under physiological conditions, DNA generally exists in intracellular solutions under siege. The interaction between the DNA elastic rod and the molecules of the solution plays a significant role in the reconfiguration of the DNA segments (Benham and Mielke, 2005). Several studies (Huang, 2011; Xiao et al., 2014; Wang et al., 2014) have shown that microequilibrium configurations of DNA chains are affected by the interaction of the DNA molecule with positive ions in a salt solution. Huang (2011) modeled the interaction using distributed traction, which is caused due to interfacial energy, in a Kirchhoff model, and characterized the folding and unfolding of DNA chains using a numerical simulation of the elastic model. However, Huang had incorrectly determined the interfacial traction on the central axis of the DNA elastic rod, which was later corrected by two different groups of researchers (Xiao et al., 2014; Wang et al., 2014) in 2014. This interaction should, in essence, be characterized by van der Waals forces or electrostatic forces and is a very critical factor in studying DNA reconfiguration.

Non-linearity in Kirchhoff equations may increase the difficulty of the calculation process. Numerical solutions of the equations can be found using many methods; the shooting method is one example (Balaeff et al., 2006). The energy balance method is valid for weakly and strongly nonlinear problems and has a very wide range of applications (Fu et al., 2011). The basic idea of the energy balance method is that when the torsional angle $\theta$ is zero, the whole energy of the system is regarded to be in the form of kinetic energy, and when $\theta$ is $\pi / 2$, the whole energy will change into potential energy. Finally, when $\theta$ is $\pi / 4$, a balance of energy is achieved between potential and kinetic energy (He, 2002), which is when the Hamiltonian of the system would be obtained, and the angular frequency can be calculated using the collocation method.

\section{MATERIAL AND METHODS}

\section{Kirchhoff's equations}

To study the configuration of DNA, the DNA is regarded as an inextensible and 
unshearable thin elastic rod with a circular cross section based on Kirchhoff's theory of elastic rod. The geometrical configuration at the microscope scale is determined by the spatial configuration of the central axis of the DNA elastic rod. In the presence of an external load $f_{i}$, the static Kirchhoff equations in a body fixed frame are as follows (Reissner, 1981; Liu, 2006):

$$
\left\{\begin{aligned}
F_{1^{\prime}}+\omega_{1} F_{2}-\omega_{3} F_{2}+f_{1} & =0 \\
F_{2^{\prime}}+\omega_{3} F_{1}-\omega_{1} F_{3}+f_{2} & =0 \\
F_{3^{\prime}}+\omega_{1} F_{2}-\omega_{2} F_{1}+f_{3} & =0 \\
M_{1^{\prime}}+\omega_{2} M_{3}-\omega_{3} M_{2}-F_{2} & =0 \\
M_{2^{\prime}}+\omega_{3} M_{1}-\omega_{1} M_{3}+F_{1} & =0 \\
M_{3^{\prime}}+\omega_{1} M_{2}-\omega_{2} M_{1} & =0
\end{aligned}\right.
$$

where the prime' is differentiation with respect to the arc length $s ; w_{i}$ denotes Darboux vector, and $F_{i}$ and $M_{i}$ are the resultant force and the corresponding moment that act at the central axis of the rod, respectively.

$$
\vec{M}=A \omega \overrightarrow{e_{1}}+B \omega_{2} \overrightarrow{e_{2}}+C\left(\omega_{3}-\omega_{3}^{0}\right) \overrightarrow{e_{3}}=E I \omega \overrightarrow{e_{1}}+E I \omega_{2} \overrightarrow{e_{2}}+G J\left(\omega_{3}-\omega_{3}^{0}\right) \overrightarrow{e_{3}} \text {. (Equation 2) }
$$

where $A=B$ and $C$ denoting the bending stiffness and twisting stiffness, respectively. $E$ denotes Young's modulus, and $G$ denotes the shear modulus. $I=p r^{4} / 4$ and $J=p r^{4} / 2$, where $r$ denotes the radius at arbitrarily given cross section of the rod. Based on the relationship between curvature $\kappa$, torsion $\tau$, and twisting angle $\chi$, the curvature-twisting vector $w_{i}$ can be expressed as follows:

$$
\omega_{1}=\kappa \sin \chi, \quad \omega_{2}=\kappa \cos \chi, \quad \omega_{3}=\tau+\chi^{\prime} . \quad \text { (Equation 3) }
$$

where $f_{i}$ refers to the interfacial traction, which characterizes the effects of the intracellular solution on the rod (Xiao et al., 2014; Wang et al., 2014).

$f_{1}=-\frac{2 \pi \sigma}{r} \frac{\omega_{2}}{\omega_{1}^{2}+\omega_{2}^{2}}\left(1-\frac{1}{\sqrt{1-r^{2}\left(\omega_{1}^{2}+\omega_{2}^{2}\right)}}\right), f_{2}=\frac{2 \pi \sigma}{r} \frac{\omega_{1}}{\omega_{1}^{2}+\omega_{2}^{2}}\left(1-\frac{1}{\sqrt{1-r^{2}\left(\omega_{1}^{2}+\omega_{2}^{2}\right)}}\right), f_{3}=0$

where $\sigma>0$ is the interfacial energy factor, which is generally a constant in a given salt solution and describes a result of the comprehensive hydrophilic and hydrophobic effect distributed along the surface of the DNA segments and relates to the solution environment (Huang, 2011). can obtain

It is known that $d w_{3} / d s=0$, that is to say that $w_{3}=$ constant. Through Equation 1, we 


$$
\frac{1}{2} A\left(\omega_{1}^{2}+\omega_{2}^{2}\right)+\frac{1}{2} C \omega_{3}^{2}+F_{3}=H=\text { constant }
$$

(Equation 4)

A complex curvature $\xi$ can be defined as $\xi=w_{1}+i w_{2}$, a complex external load can be defined as $f=f_{1}+i f_{2}$, and a complex elastic force can be defined as $F=F_{1}+i F_{2}$. From the fourth and fifth equations of (1), we can get

$$
\frac{d \xi}{d s}+i\left(\omega_{3} F-\frac{C}{A}\left(\omega_{3}-\omega_{3}^{0}\right) \xi\right)+i F=0
$$

Likewise, from the first and second equations of (1), we can obtain

$$
\frac{d F}{d s}+i \omega_{3} F-i\left(H-\frac{1}{2}|\xi|^{2}\right) \xi+i \frac{2 \pi \sigma}{r}\left(1-\frac{1}{\sqrt{1-r^{2}|\xi|^{2}}}\right) \frac{\xi}{|\xi|}=0 . \text { (Equation 6) }
$$

We can then eliminate $F(s)$ from Equations 5 and 6, to obtain

$$
\frac{d^{2} \xi}{d s^{2}}+i a \frac{d \xi}{d s}-b \xi+\frac{1}{2}|\xi|^{2} \xi+\frac{2 \pi \sigma}{r|\xi|^{2}}\left(1-\frac{1}{\sqrt{1-r^{2}|\xi|^{2}}}\right)=0 . \quad \text { (Equation 7) }
$$

where $a=2 \omega_{3}-C\left(\omega_{3}-\omega_{3}^{0}\right) / A, b=\omega_{3}^{2}-C \omega_{3}\left(\omega_{3}-\omega_{3}^{0}\right) / A+H$.

Now, we recall Equation 3, that is $\xi=\kappa(s) \exp [i(\pi / 2-\chi)]$ and $\frac{d \xi}{d s}=\omega_{3}-\tau$. By substituting these two equations into Equation 7, and then by separating the real part and imaginary part, we can get

$$
\left\{\begin{array}{c}
\frac{d^{2} \kappa}{d s^{2}}-\kappa\left(\tau-\frac{m}{2}\right)^{2}-c \kappa+\frac{1}{2} \kappa^{3}+\frac{2 \pi \sigma_{1}}{r \kappa}\left(1-\frac{1}{\sqrt{1-r^{2} \kappa^{2}}}\right)=0 . \\
\kappa \frac{d \tau}{d s}+2 \frac{d \kappa}{d s}\left(\tau-\frac{m}{2}\right)=0
\end{array}\right.
$$

where $m=\omega_{3}-\frac{a}{2}, c=b-\frac{a^{2}}{4}$ and $\sigma_{1}=\frac{\sigma}{A}$. It is obvious that $\kappa^{2}\left(\tau-\frac{m}{2}\right)=J=$ constant. Thus, Equation 8 can be written as

$$
\frac{d^{2} \kappa}{d s^{2}}-\frac{J^{2}}{\kappa^{3}}-c \kappa+\frac{1}{2} \kappa^{3}+\frac{2 \pi \sigma_{1}}{r \kappa}\left(1-\frac{1}{\sqrt{1-r^{2} \kappa^{2}}}\right)=0
$$


It is clear that Equation 9 is a nonlinear differential equation, which characterizes the conformation of the DNA rod. The centerline of the rod is solely determined, up to a rigid body motion, by its curvature $\kappa(s)$ and torsion $\tau(s)$. Therefore, one can obtain the conformation of the DNA rod by solving Equation 9.

\section{Energy balance method}

\section{Introduction of the energy balance method}

Consider the nonlinear equation in the following form (He, 2002; Ganji, et al., 2009; Fu et al., 2011):

$$
\frac{d^{2} u}{d x^{2}}+f(u(x))=0, u(0)=D, u^{\prime}(0)=0
$$

where $u$ is the function of the variable $x$.

The following Hamiltonian is established for the above equation using the semiinverse method

$$
H=\frac{1}{2} u^{\prime 2}+F(u)=F(D)
$$

where $F(u)$ is the antiderivative of $f(u)$.

The trial function is

$$
u(x)=D \cos \varpi x
$$

which can then be employed to determine the angular frequency. The following residual is consequently obtained as

$$
R(x)=\frac{1}{2} \varpi^{2} D^{2} \sin ^{2} \varpi x+F(D \cos \varpi x)-F(D)=0
$$

Using the collocation method at $\varpi x \rightarrow \pi / 4$, one can obtain

$$
\varpi_{E B M}=\sqrt{\frac{4(F(D)-F(D \sqrt{2} / 2))}{D^{2}}}
$$

\section{Application of the energy balance method}

We now use the energy balance method to solve the conformation of the DNA elastic rod. Take Equation 9 as the governing equation. Its variational formulation is

$$
J(\kappa)=\int_{0}^{s}\left(-\frac{1}{2} \kappa^{\prime 2}+\frac{J^{2}}{2 \kappa^{2}}-\frac{c}{2} \kappa^{2}+\frac{1}{8} \kappa^{4}+2 \pi \sigma_{1} \ln \left(1+\sqrt{1-r^{2} \kappa^{2}}\right)\right) d s
$$


Its Hamiltonian, therefore, can be written in the form

$$
H=\frac{J^{2}}{2 D^{2}}-\frac{c}{2} D^{2}+\frac{1}{8} D^{4}+2 \pi \sigma_{1} \ln \left(1+\sqrt{1-r^{2} D^{2}}\right)
$$

Substituting Equation 10 into the above equation, we can obtain

$$
\begin{aligned}
R= & \frac{1}{2}(D \varpi \sin \varpi s)^{2}+\frac{J^{2}}{2}\left(\frac{1}{(D \cos \varpi s)^{2}}-\frac{1}{D^{2}}\right)-\frac{c}{2}\left((D \cos \varpi s)^{2}-D^{2}\right) \\
& +\frac{1}{8}\left((D \cos \varpi s)^{4}-D^{4}\right)+2 \pi \sigma_{1} \ln \left(\frac{1+\sqrt{1-r^{2}(D \cos \varpi s)^{2}}}{1+\sqrt{1-r^{2} D^{2}}}\right)
\end{aligned}
$$

We can determine the frequency $\varpi=\frac{\sqrt{2}}{D \sin \varpi s} \sqrt{P}$ where

$$
\begin{aligned}
P= & -\frac{J^{2}}{2}\left(\frac{1}{(D \cos \varpi s)^{2}}-\frac{1}{D^{2}}\right)+\frac{c}{2}\left((D \cos \varpi s)^{2}-D^{2}\right)-\frac{1}{8}\left((D \cos \varpi s)^{4}-D^{4}\right) \\
& -2 \pi \sigma_{1} \ln \left(\frac{1+\sqrt{1-r^{2}(D \cos \varpi s)^{2}}}{1+\sqrt{1-r^{2} D^{2}}}\right) .
\end{aligned}
$$

If we collocate at $\varpi s=\pi / 4$, we can obtain

$$
\varpi_{E B M}=\frac{2}{D} \sqrt{-\frac{J^{2}}{2 D^{2}}-\frac{c}{4} D^{2}+\frac{3}{32} D^{4}+2 \pi \sigma_{1} \ln \left(\frac{1+\sqrt{1-r^{2} D^{2}}}{1+\sqrt{1-\frac{1}{2} r^{2} D^{2}}}\right)}
$$

Substituting $\varpi_{E B M}$ into Equation 10 yields

$$
\kappa(s)=D \cos \left(\frac{2}{D} \sqrt{\left.-\frac{J^{2}}{2 D^{2}}-\frac{c}{4} D^{2}+\frac{3}{32} D^{4}+2 \pi \sigma_{1} \ln \left(\frac{1+\sqrt{1-r^{2} D^{2}}}{1+\sqrt{1-\frac{1}{2} r^{2} D^{2}}}\right) s\right)}\right.
$$

Figure 1 shows the numerical solutions obtained using both the energy balance method and the fourth-order Runge-Kutta method. The results show that the periodical solutions of (Equation 9) obtaining using the energy balance method maintain high accuracy. Thus, the approximate variational method, the energy balance method, is a powerful tool for dealing with nonlinear behaviors, and can provide high accuracy solutions for smooth nonlinear systems. (Equation 11), which is obtained using the energy balance method, will provide great help for our next research on the configuration of the DNA segments. Using the Frenet formulae, the configurations of the rod can be obtained, which are shown in Figure 2. The results show that 
the reconfiguration of the DNA elastic rod was owing to the interaction between the interfacial traction and the elastic property, which has also been previously reported by Xiao et al. (2014).

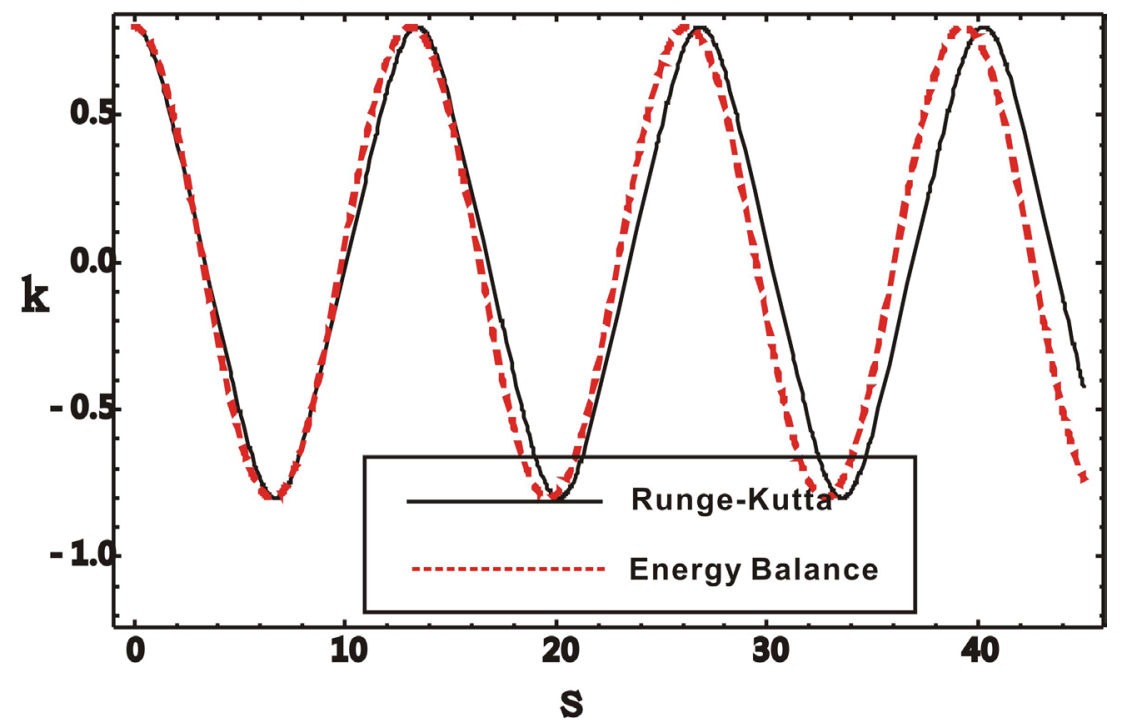

Figure 1. Comparison between the energy balance method and the fourth-order Runge-Kutta method with $\mathrm{s}_{1}=1 \mathrm{x}$ $10^{-5}, \mathrm{r}=1, \mathrm{~J}=0, \mathrm{c}=0.01$ and $\mathrm{D}=0.8$.
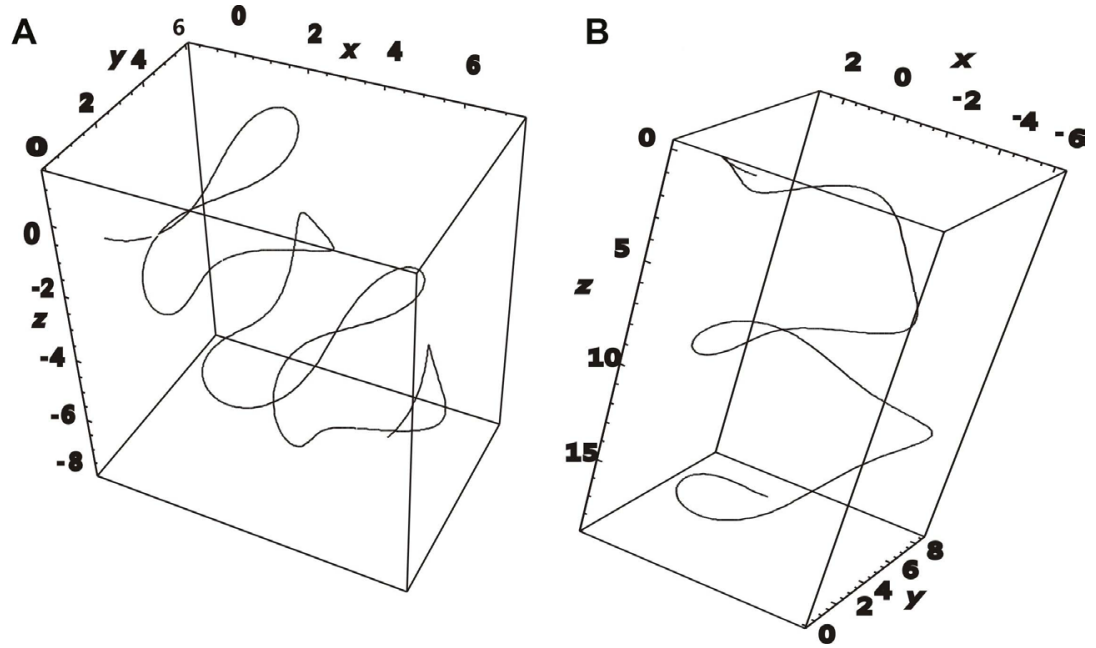

Figure 2. DNA conformation with $\mathrm{s}_{1}=1 \times 10^{-5}, \mathrm{r}=1, \mathrm{~J}=0, \mathrm{c}=0.01, \mathrm{D}=0.8(\mathbf{A})$ and $\mathrm{D}=0.5(\mathbf{B})$.

A kink is characterized by a piece of a filament with a high curvature $\kappa$ (or small radius of curvature, $R=1 / \kappa$ ) (Alexandre et al., 2006). The Kirchhoff approach is not appropriate for modeling kinks in rods because, in the derivation of Kirchhoff equations, it is assumed that the centerline radius of curvature of the rod is much larger than the cross-sectional radius (Huang, 2011). However, Zhao et al. (1998) used the Kirchhoff rod model to find that the spontaneous 
curvature has a great influence on the stability of kinked DNA. Zhou and Liu (2013) later also analyzed the stability of kinked DNA with the second variation of the generalized elastic free energy. With the limits of validity of the Kirchhoff model, Equation 11 shows that some pieces of a rod with a small interfacial energy factor may lead to equilibrium configuration with a large curvature in the case of an initial given curvature. Thus, our results can help explain the onset of the formation of kinks.

In a more recent study, Xiao et al. (2014) accurately obtained the interfacial energy factor in terms of the concentration of the salt solution using the following formula:

$$
\sigma=\sigma_{0}-\Gamma_{\max } R T \log (1+K \eta)
$$

(Equation 12)

where $s_{0}$ is the interfacial tension of pure solvent, $\Gamma_{\max }$ is the maximum concentration when the solution arrives at its saturation state, $\eta$ is the concentration of the solution, $T$ denotes the temperature, and $\mathrm{K}$ is the absorption constant. It is clear that the interfacial energy factor is a decreasing function of the concentration. From Equations 11 and 12, at some point in the rod, we can easily find that the DNA segments become kinked when the concentration of the $\mathrm{Zn}^{2+}$ ions rises above a critical value, which is fairly consistent with the results of Han et al. (1997).

\section{CONCLUSION}

Based on the classical elasticity theory, in this study, the DNA is regarded as a thin elastic rod in the presence of distributed interfacial traction, which characterizes the effect of intracellular solutions on DNA reconfiguration. Further, the energy balance method has been well applied to analyze a nonlinear equation, which describes the conformation of the DNA elastic rod. In addition, the analytic approximation of this nonlinear problem has high accuracy and provides a relationship between the interfacial energy factor and the curvature. Thus, our results can give a new insight into the phenomenon of kink formation in DNA segments.

\section{Conflicts of interest}

The authors declare no conflict of interest.

\section{ACKNOWLEDGMENTS}

Research supported by the National Nature Science Foundation of China (\#11372210), Key scientific research projects of colleges and Universities of Henan Province (\#16A130004 and \#16A110028) and the Tianjin Research Program of Application Foundation and Advanced Technology (\#12JCZDJC 28000). This support is greatly appreciated.

\section{REFERENCES}

Alexandre F, Fonseca D and Malta CP (2006). Circular and helical equilibrium solutions of inhomogeneous rods. arXiv preprint physics. 0607132.

Balaeff A, Mahadevan L and Schulten K (2006). Modeling DNA loops using the theory of elasticity. Phys. Rev. E Stat. Nonlin. Soft Matter Phys. 73: 031919. http://dx.doi.org/10.1103/PhysRevE.73.031919

Benham CJ and Mielke SP (2005). DNA mechanics. Аnnu. Rev. Biomed. Eng. 7: 21-53. http://dx.doi.org/10.1146/annurev. bioeng.6.062403.132016 
Bouchiat C and Mezard M (1998). Elasticity model of a supercoiled DNA molecule. Phys. Rev. Lett. 80: 1556. http:// dx.doi.org/10.1103/PhysRevLett.80.1556

Elf J, Li GW and Xie XS (2007). Probing transcription factor dynamics at the single-molecule level in a living cell. Science 316: 1191-1194. http://dx.doi.org/10.1126/science.1141967

$\mathrm{Fu} \mathrm{Y,} \mathrm{Zhang} \mathrm{J} \mathrm{and} \mathrm{Wan} \mathrm{L} \mathrm{(2011).} \mathrm{Application} \mathrm{of} \mathrm{the} \mathrm{energy} \mathrm{balance} \mathrm{method} \mathrm{to} \mathrm{a} \mathrm{nonlinear} \mathrm{oscillator} \mathrm{arising} \mathrm{in}$ the microelectrome-chanical system (MEMS). Curr. Appl. Phys. 11: 482-485. http://dx.doi.org/10.1016/j. cap.2010.08.037

Ganji SS, Ganji DD and Karimpour S (2009). Periodic solution for strongly nonlinear vibration systems by He's energy balance method. Int. J. Mod. Phys. B 106: 79-92.

Goldstein RE and Langer SA (1995). Nonlinear dynamics of stiff polymers. Phys. Rev. Lett. 75: 1094-1097. http://dx.doi. org/10.1103/PhysRevLett.75.1094

Halford SE and Marko JF (2004). How do site-specific DNA-binding proteins find their targets? Nucleic Acids Res. 32: 3040-3052. http://dx.doi.org/10.1093/nar/gkh624

Han W, Dlakic M, Zhu YJ, Lindsay SM, et al. (1997). Strained DNA is kinked by low concentrations of Zn2+. Proc. Natl. Acad. Sci. USA 94: 10565-10570. http://dx.doi.org/10.1073/pnas.94.20.10565

He JH (2002). Preliminary report on the energy balance for nonlinear oscillations. Mech. Res. Commun. 29: 107-111. http://dx.doi.org/10.1016/S0093-6413(02)00237-9

Huang Z (2011). Modulating DNA configuration by interfacial traction: an elastic rod model to characterize DNA folding and unfolding. J. Biol. Phys. 37: 79-90. http://dx.doi.org/10.1007/s10867-010-9200-x

Liu YZ (2006). Nonlinear Mechanics of Thin Elastic Rod: Theoretical Basis of Mechanical Model of DNA (Tsinghua Press, Beijing) 42.

Luger K, Mäder AW, Richmond RK, Sargent DF, et al. (1997). Crystal structure of the nucleosome core particle at 2.8 A resolution. Nature 389: 251-260. http://dx.doi.org/10.1038/38444

Reissner E (1981). On finite deformations of space-curved beams. Z. Angew. Math. Phys. 32: 734-744. http://dx.doi. org/10.1007/BF00946983

Wang Y, Zhang Q and Wang W (2014). Comment on "Modulating DNA configuration by interfacial traction: an elastic rod model to characterize DNA folding and unfolding". J. Biol. Phys. 40: 259-266. http://dx.doi.org/10.1007/s10867$\underline{014-9345-0}$

Xiao Y, Huang Z and Wang S (2014). An elastic rod model to evaluate effects of ionic concentration on equilibrium configuration of DNA in salt solution. J. Biol. Phys. 40:179-192. http://dx.doi.org/10.1007/s10867-014-9344-1

Yavari M (2014). The study of the free energy model $F=\sqrt{\tau}$ for the biopolymer structures. Eur. Phys. J. Plus. 129: 1-7.

Zhao W, Zhou H and Ou-Yang ZC (1998). Kink instability in circular DNA studied as Helfrich chiral chain. Phy. Rev. E. 58: 8040. http://dx.doi.org/10.1103/PhysRevE.58.8040

Zhou XH and Liu JL (2013). Stability analysis of kinked DNA with generalized rod model. Physica E 47: 152-156. http:// dx.doi.org/10.1016/j.physe.2012.10.037 